\title{
$=\mathrm{T} R A M A=$
}

\section{A NANOSSINTAXE NOS PROCESSOS DE LEXICALIZAÇÃO NO JARGÃO DOS “GAMERS”}

\author{
MANOEL RIGEL DIAS (UFPR) ${ }^{1}$ \\ https://orcid.org/0000-0002-4663-0468 \\ TERESA CRISTINA WACHOWICZ (UFPR)² \\ https://orcid.org/0000-0003-2917-4031
}

\begin{abstract}
RESUMO: A comunidade "gamer" possui uma linguagem especial para tratar das questões que os jogos apresentam. Esse jargão contém novas palavras que irão descrever aquilo que acontece no jogo, o mapa, as estratégias usadas e os elementos presentes. Essa pesquisa objetivou analisar a semântica usada nessas criações lexicais, utilizando os dados retirados do chat de League of Legends. Para isso, mapeamos a literatura sobre o fenômeno de lexicalização, começando pela perspectiva decomposicionalista de Jackendoff (1990), que afirma a existência de primitivos semânticos básicos que atuam em todas as línguas. Aprimorando essa visão com a proposta de Von Fintel \& Mathewson (2008) sobre blocos derivacionais que atuariam como um sistema de hierarquia entre os traços semânticos, esses regidos pela sintaxe. Finalmente, percorrendo pelo tratamento dado pela nanossintaxe (RAMCHAND $(2008,2017)$ e PANCHEVA $(2009))$, esse mais inclinado a teoria de Jackendoff, que observa que a estrutura lexical é amalgamada com a estrutura da sintaxe, sendo o léxico o processo inicial da sentença. Com essa estrutura argumentativa, foi possível analisarmos os dados. Concluímos que maior parte das novas palavras apresenta traços de PLACE e PATH, devido à natureza logística do jogo, e que a nanossintaxe apresenta melhor forma de descrever hierarquicamente a lexicalização dessa estrutura.
\end{abstract}

PALAVRAS-CHAVE: Processos de Lexicalização. League of Legends. Nanossintaxe.

ABSTRACT: The "gamer" community has a special language to address the issues that games presents. This jargon contains new words that will describe what happens in the game, the map, the strategies used and the elements present. This research aimed to analyze the semantics used in these lexical creations, using the data taken from the League of Legends chat. For this, we mapped the literature on the phenomenon of lexicalization, starting with the decompositionalist perspective of Jackendoff (1990), who affirms the existence of basic primitive semantics that work in all languages. Improving this view with the proposal of Von Fintel \& Mathewson (2008) on derivational blocks that would act as a hierarchy system between the semantic features, those governed by syntax. Finally, going through the treatment given by nanosyntax (RAMCHAND $(2008,2017)$ e PANCHEVA (2009)), this one more inclined to Jackendoff's theory, which observes that the lexical structure is amalgamated with the structure of the syntax, the lexicon being the initial process of the sentence. With this argumentative structure, it was possible to analyze the data. We conclude that most of the new words have traces of PLACE and PATH, due to the logistical nature of the game, and that nanosyntax presents a better way of hierarchically describing the lexicalization of this structure.

KEYWORDS: Lexicalization Processes. League of Legends. Nanosyntax.

\footnotetext{
${ }^{1}$ Mestrando em Estudos Linguísticos pela Universidade Federal do Paraná (UFPR) e atua na área de Morfologia. E-mail: manoeldias@alunos.utfpr.edu.br

2 Docente do Departamento de Literatura e Linguística da Universidade Federal do Paraná (UFPR). E-mail: tecacw@gmail.com
} 


\section{$=$ TRAMA $=$}

\section{INTRODUÇÃO}

Para quem mantém algum contato com jovens que gostam de jogar videogame, é muito comum se surpreender com uma linguagem diferenciada (entre eles), principalmente no uso de um léxico específico, que - claro - legitima politicamente o grupo. Expressões como "deu hate", "tá gankando", "olha esse bot" tornam-se semanticamente inacessíveis aos ouvidos de adultos.

No entanto, para além de uma sociologia do léxico, há um movimento linguístico nesses dados, que envolve lexicalização e ambientação gramatical. Nosso objetivo neste artigo é analisar a semântica que está por trás da criação lexical.

Nesse sentido, lançamos mão de algumas vertentes da semântica das línguas naturais que tratam o fenômeno de lexicalização. Inicialmente, a semântica conceitual (JACKENDOFF, 1990; 2012) nos fornece os primitivos relevantes à gramática (PINKER, 1989, LANGACKER, 1993, WUNDERLICH, 1997), tais como PLACE, PATH etc., assumindo um processo composicional em que a primeira fase da derivação (VON FINTEL \& MATHEWSON, 2008) envolve a conceptualização do evento - o lugar da estrutura lexical - exploramos a proposta nanossintática de Ramchand (2008, 2017), e Pancheva (2009) para descrever a organização hierárquica de traços semânticos que fundamentam as opções lexicais dos 'gamers'.

$\mathrm{Na}$ seção 1, apresentamos um quadro de verbos, organizado segundo suas interpretações aspectuais e decomposicionais. Concluímos que há uma forte tendência de os 'gamers' criarem verbos selecionando traços da natureza localística do jogo (GRUBER, 1965): PATH e PLACE. Na seção 2, fundamentamos a análise a partir dos primitivos conceituais relevantes à gramática, na defesa das zonas de derivação (simplificadamente: zona lexical, zona gramatical, zona pragmática), que se iniciam pelo ambiente mais baixo da estrutura: o VP, o evento, ou a zona de conteúdo lexical. A seção 3 lista algumas considerações e/ou ressalvas, no sentido de apontar discussões futuras.

\section{OS DADOS}

League of Legends, ou LOL, como é chamado pelos seus jogadores, é um jogo virtual do gênero MOBA (Multiplayer Online Battle Arena) criado em 2009 pela desenvolvedora Riot Games. No jogo, 10 jogadores são divididos dentro de um mapa em 2 times de 5. O objetivo é destruir o Nexus inimigo.

Para esse fim, os jogadores (também chamados de invocadores), devem escolher entre os mais de 150 personagens jogáveis e atuar 4 em rotas principais: a rota do topo (top), meio (mid), selva (jungle) e inferior (bot). As rotas são o único caminho até o Nexus, porém elas são protegidas por torres. Os campeões (o nome dado aos personagens jogáveis) possuem habilidades únicas e podem comprar itens que os ajudem a enfrentar o outro time.

Como em outros MOBAs, a estratégia, comunicação e o trabalho em equipe são essenciais. Dessa forma, é necessária uma linguagem que possa transmitir essas informações de maneira ágil e prática, para que os jogadores consigam vencer.

Dias \& Rutiquewiski (2019) analisaram as neologias usadas por esse jargão e contabilizaram vários dados retirados do chat usado pelos jogadores. Os pesquisadores verificaram a criação de palavras que possuem uma base formada por um empréstimo que pode passar por processos de derivação para se tornarem verbo. Para esta pesquisa, foi usado o banco de dados produzido por eles.

Especificamente em relação ao conteúdo aspectual desses novos verbos, é possível aplicar uma combinatória de traços ( \pm DURATIVO, \pm HOMOGÊNEO, \pm AGENTIVO), definidores das classes aspectuais clássicas (estados, atividade, accomplishments, achievements) (VENDLER, 1967, SMITH, 1997, BERTINETTO, 2001). Optamos aqui por apresentar esta análise em quatro verbos visivelmente produtivos no jargão dos gamers: 


\section{$=$ TRAMA $=$}

Quadro 1 - Verbos encontrados e suas classes aspectuais.

\begin{tabular}{|c|c|c|}
\hline Gankar - Emboscada em outra lane & Atividade & $\begin{array}{l}+ \text { DURATIVO } \\
+ \text { HOMOGENEO } \\
+ \text { AGENTIVO }\end{array}$ \\
\hline $\begin{array}{l}\text { Caitar - Manter uma distância segura entre o } \\
\text { campeão e o oponente, enquanto você recua e, } \\
\text { ao mesmo tempo, ataca durante o avanço } \\
\text { inimigo. }\end{array}$ & Achievement & $\begin{array}{l}\text { - DURATIVO } \\
\text { - HOMOGÊNEO } \\
\text { - AGENTIVO }\end{array}$ \\
\hline Solar - Abater sem a ajuda de outros jogadores. & Atividade & $\begin{array}{l}+ \text { DURATIVO } \\
+ \text { HOMOGENEO } \\
+ \text { AGENTIVO }\end{array}$ \\
\hline Flashar - Usar o feitiço Flash. & Achievement & $\begin{array}{l}\text { - DURATIVO } \\
\text { - HOMOGÊNEO } \\
\text { + AGENTIVO }\end{array}$ \\
\hline
\end{tabular}

Fonte: Autoria Própria.

Os contextos de uso estão alocados nos dados do corpus descrito acima:

Gankar: "(Swain): ta gankando kayn?" (Dado 1);

"(Syndra) Ivl 6 ou 7 eu te ganko ornn" (Dado 32).

Caitar: "- A Vayne é muito boa para caitar a Shyvanna [...] Tem uma mobilidade boa para caitar essa Shyvanna, para caitar esse Tahm Kemch" (Dado 9)

Solar: "(Fiora) começa bot qyina

(Fiora) se tu nao conseguir solar" (Dado 39)

Flashar: "(Ezreal) boa pyke

(Ezreal) flashou

(Ezreal) tnc "(Dado 40)

É interessante perceber que mesmo com uma criação nova, o jargão continua a obedecer e a pertencer a determinados processos semânticos presentes supostamente em todas as línguas, como é o caso de um conteúdo lexical temporalmente configurado, como é o caso das classes aspectuais dos verbos (VONFINTEL \& MATHEWSON, 2008). No concreto, o jogador 'lexicaliza' diversos traços semânticos nesses verbos, já que, dessa forma, podem se comunicar melhor dentro do jogo.

Mas há uma particularidade no jargão dos gamers que diz respeito à motivação situacional em que esses verbos surgem. Trata-se de um jogo que acontece em um espaço em que os jogadores atuam em rotas alternativas para conseguirem seus objetivos sob uma determinada maneira. Logo, esses verbos, para além de exibirem traços de aspecto lexical, também lexicalizam traços da ordem do movimento espacialmente localizado: o movimento (MOTION), o lugar (PLACE), a trajetória, ou rota (PATH), a maneira do movimento (MANNER).

O verbo 'solar' por exemplo, é uma atividade e parece lexicalizar uma maneira de movimento. Já 'flashar', um achievement, lexicaliza apenas a maneira, já que não prevê o movimento do jogador. Em um esboço de representação já sinalizando uma semântica 


\section{$=$ TRAMA $=$}

conceitual de traços (JACKENDOFF, 1990, 2012), podemos visualizar nesses verbos os seguintes traços:

\begin{tabular}{|c|c|c|}
\hline Solar & $\Rightarrow$ & $\begin{array}{r}{[\text { MOTION, }} \\
\text { MANNER }]\end{array}$ \\
\hline flashar & $\Rightarrow$ & {$[$ MANNER $]$} \\
\hline
\end{tabular}

Com a finalidade de analisar mais atentamente os verbos que lexicalizam informações espaciais, como o lugar e a trajetória, recortaremos os verbos caitar e gankar, como representantes de um fenômeno de lexicalização produtivo no jargão em tela:

\begin{tabular}{|c|c|c|}
\hline caitar & $\Rightarrow$ & {$[\mathrm{PLACE}]$} \\
\hline gankar & $\Rightarrow$ & {$[\mathrm{PATH}$, PLACE $]$} \\
\hline
\end{tabular}

De fato, alguns traços discutidos nesta seção parecem sobrepor intuições semânticas. Se 'solar' é uma atividade, é previsível que haja movimento, possivelmente de uma determinada maneira. Os traços DURATIVO e AGENTIVO podem, portanto, sobrepor-se ao traço de movimento - MOTION. A opção pelo tipo de notação de traços semânticos vai depender, obviamente, do recorte do modelo de representação potencialmente mais interessante para fins de análise da lexicalização. Nesse sentido, abordaremos mais detalhadamente a proposta da semântica conceitual de Jackendoff (...), estendida à proposta de representação lexical já incorporada com estrutura sintática, defendida pela Nanossintaxe (RAMCHAND, 2008 e PANCHEVA, 2009). É esse percurso teórico que desenvolvemos na seção seguinte.

\section{A SEMÂNTICA NOS DADOS}

Se a grande questão é investigar os motivos de processos de lexicalização no jargão dos 'gamers', e se estamos assumindo uma perspectiva semântica na investigação, acreditamos que existam traços, primitivos, ou conceitos básicos, que estão codificados no item 'novo'. Intuitivamente, percebemos o significado de 'lugar' na expressão "mid izi” (posição fácil), ou o significado de 'trajetória' em "bom gank" (boa emboscada).

Do ponto de vista teórico, duas questões: i. Podemos prever alguns primitivos semânticos que condicionariam esses processos de lexicalização? ii. Ou mais ousadamente, esses traços estariam dispostos em alguma organização estrutural para condicionar a criatividade lexical?

Para responder à primeira pergunta, recorremos historicamente à perspectiva decomposicionalista da semântica gerativa, especialmente na figura de Jackendoff (1990, 2012), que é o nome mais produtivo dessa tradição, e que trouxe os trabalhos pioneiros. Seguindo o conceito de nível pré-linguístico, de Gruber (1965), concebe-se a semântica decomposicionista como uma etapa anterior à realização da frase, na opção de representação de uma estrutura conceitual lexical (ou Lexical Conceptual Structure (LCS)), hoje figurando como base teórica de estudos de Levin (1993), Levin \& Rappaport Hovav (1998, 2005), e Cançado et. al (2013), aqui no Brasil.

Segundo Jackendoff (1990), os traços semânticos primitivos, que formam o conjunto de conceitos básicos ou categorias ontológicas básicas, podem inicialmente ser os seguintes: THING, EVENT, ACTION, PLACE, PATH, PROPERTY, MANNER, AMOUNT ${ }^{3}$. O argumento empírico para essa lista é universalista; são traços semânticos que atuam supostamente em

\footnotetext{
${ }^{3}$ Já é consenso na literatura em semântica conceitual, lexical, ou decomposicionalista, que os traços primitivos sejam escritos em caixa alta.
} 


\section{$=$ TRAMA $=$}

todas as línguas. Como argumento linguístico, o autor se utiliza de um comportamento produtivo de perguntas que correspondentes aos traços: O que você comprou?; O que aconteceu?; O que você fez?; Onde está meu casaco?; Por onde você andou?; O que está por trás disso?; Como você cozinhou os ovos?; Que tamanho tem o peixe?, respectivamente ${ }^{4}$ (p. 53).

Para além das categorias primitivas, existem funções que expandem composicionalmente esses constituintes dentro do significado lexical: GO, ACT, HAVE, BE. Outro elemento importante: a relação CAUSE, que seleciona dois eventos. Tomando um evento de movimento, como na sentença (1), sua estrutura conceitual estaria representada em (1'):

(1) João foi ao mercado.

(1') EVENTO $\rightarrow$ [EVENT GO (THING, PATH)]

Outro exemplo ficaria por conta de um caso de ambiguidade, como 'rolar', um verbo de maneira de movimento (2). No PB, a alternância de (2a) para (2b) ilustra o fenômeno de causativização de verbo inergativo. A proposta de estrutura conceitual gera então itens lexicais diferentes, como em (2') (RAMMÉ, 2012):

(2) a. A bola rolou 1 no campo

b. João rolou 2 a bola no campo

(2') a. Rolar 1 : EVENTO $\rightarrow$ [EVENT GO (THING, PLACE [in THING] , MANNER]

b. Rolar $2:$ EVENTO $\rightarrow$ [EVENT ACT (THING, EVENT [GO (THING, PLACE [in THING] , MANNER]

O princípio central da teoria prevê que todos os lugares da estrutura conceitual podem ser preenchidos por um traço: Princípio da variável lexical: "A variable in the structure of a lexical item must be capable of being filled by a conceptual constituent" (1990, p. 185).

Mas é inevitável que surjam outras e consequentes discussões sobre a natureza e a especificação dessas categorias. Quanto à natureza, Jackendoff resgata a própria noção de 'sentido' de Frege (2009), dissociada das categorias lógicas básicas de um sistema formal, que incluiria, grosso modo, apenas predicados e argumentos aos quais os predicados se aplicam. Recuperando o sentido, Jackendoff toma como consequência o tratamento cognitivista e universalista dos traços semânticos básicos.

Quanto à especificação dessas categorias, a grande questão passa a ser: quais os traços são relevantes à estrutura conceitual? Mais ainda, quais são condicionantes à gramática, funcionando como restrições? A resposta sugere uma dissociação básica entre uma semântica que entra na gramática e uma semântica que figura em conhecimento de mundo, ou conhecimento enciclopédico. Pinker (1989) formula hipóteses para a sistematização dos traços. A semântica da estrutura é regida pela Hipótese do subsistema gramaticalmente relevante; e a semântica de conhecimento enciclopédico é regida por condições externas à gramática, na Hipótese de representação conceitual irrestrita (ibidem, p. 166). Numa terminologia mais intuitiva: estrutura semântica e conteúdo semântico (SAEED, 2003).

No entanto, a partir do significado decomposicional dos itens lexicais e do conjunto de traços gramaticalmente relevantes, encontramos um problema: como esses itens lexicais entram na derivação? É aqui também que podemos retomar a segunda pergunta desta seção:

\footnotetext{
${ }^{4}$ Tradução adaptada de "What did you buy?; What happened next?; What did you do?; Where is my coat?; How did you cook the eggs?; How long was the fish?"
} 


\section{$=\mathrm{T} R A M A=$}

esses traços estariam dispostos em alguma organização estrutural hierárquica para condicionar a criatividade lexical?

Inicialmente, uma perspectiva semântica composicional pode sinalizar o lugar da derivação sintática em que essas lexicalizações acontecem. Em Von Fintel \& Mathewson (2008), encontramos a proposta dos blocos derivacionais, que sinalizariam uma tentativa de organizar os lugares dos universais semânticos:

Figura 1: Os 'building blocks' propostos como os lugares dos universais semânticos.

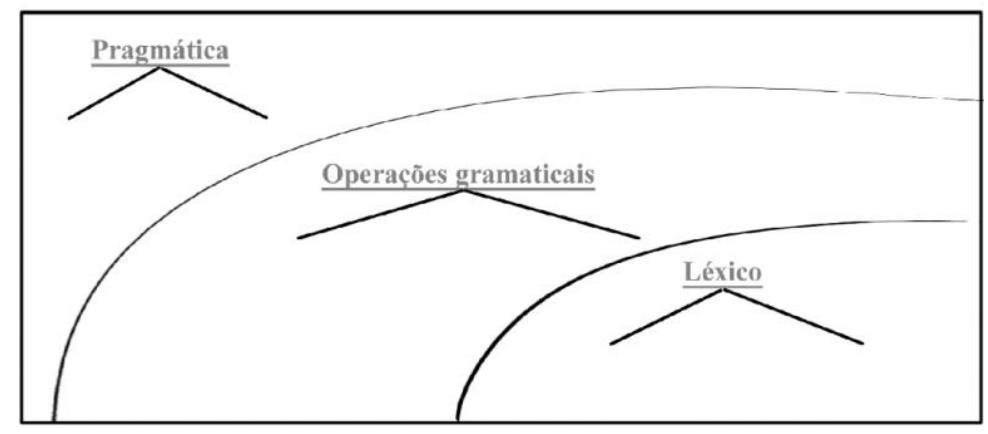

Fonte: VonFintel \& Mathewson (2008).

Ou seja, as línguas parecem exibir um comportamento semântico comum, que estaria alocado na sintaxe, em uma divisão geral prevista na derivação bottom-up, que compreende as atividades do léxico, das operações gramaticais e do contexto pragmático. Nos termos dos autores, o inventário do léxico, os mecanismos de operações gramaticais e os mecanismos pragmáticos:

Nós novamente encontramos evidências que as línguas podem compartilham aspectos fundamentais de significado, enquanto diferem na lexicalização de algumas distinções ou na estratégia sintática usada para alcançar uma semântica similar... suspeitamos fortemente que existam blocos de construção a serem encontrados (VonFINTEL \& MATHEWSON, 2008, p. 171-172)

As classes aspectuais vendlerianas seriam um fenômeno semântico universal observável na primeira zona, a do léxico:

O fato que a semântica dos accomplishments e outras classes aspectuais podem variar de língua para língua sugere que o que é universal pode não ser as classes, mas talvez o menor bloco de construção de que a estrutura de evento é composta (idem, p. 154) .

Parece óbvio. E de fato, essa composição de zonas é assumida por vários outros autores (SVENONIOUS \& RAMCHAND, 2014; RAMCHAND, 2017, etc.). Então, para vislumbrarmos o processo de lexicalização e seus primitivos, inclusive os da natureza de lugar (PLACE) e trajetória (PATH), temos que destrinchar esta primeira zona - a do léxico.

\footnotetext{
5 "We again find evidence that languages may share fundamental aspects of meaning, while differing in the lexicalization of certain distinctions or in the syntactic means they use to achieve a similar semantics... we strongly suspect that there are common semantic building blocks to be found" (Traduções das citações pelos autores).

6 The fact that the semantic of 'accomplishments' and other aspectual classes can vary from language to language suggests that what is universal may not be the classes themselves, but perhaps the smaller building blocks from which event structures are composed...
} 


\section{$=$ TRAMA $=$}

Numa perspectiva decomposicionalista, como vimos acima, cada item lexical entra na sintaxe já com todas as informações estruturadas. O verbo rolar, ambíguo, precisa de duas entradas. No entanto, do ponto de vista da combinação com a sintaxe, seu conteúdo semântico torna-se muito pesado e sua estrutura em nada conversa com a sintaxe via spell-out.

Na Nanossintaxe, o item lexical já vem com uma estrutura sintática, compartilhada e amalgamada à sintaxe da sentença. Nesse sentido, não há mais razão para separar os módulos da sintaxe e do léxico, pois ambos têm o mesmo comportamento, ou obedecem aos mesmos princípios. Melhor ainda, ambos respondem a um preceito cognitivista da recursividade, que na sintaxe gerativa traduz-se por Merge, e na semântica formal, por lambda ( $\lambda$ ) (KRIVINE, 2018).

No que foi exposto acima, a Nanossintaxe associa semântica, sintaxe e cognição na mesma estrutura - recursiva, binária, derivacional. E é o léxico que fará esse elo inicial na estruturação da sentença. A generalização que pretendemos com nossos dados parte daqui. Mas, para chegar a eles, precisamos percorrer os princípios gerais da teoria, expostos em programa teórico de Starke (2009).

Se o léxico tem estrutura, ele não é uma unidade indivisível, contrariando uma visão sintática tradicional de que o léxico é um conjunto de itens que preenchem nós terminais da estrutura. Por outro lado, isso responde a um preceito cartográfico de multiplicação de nós (CINQUE, 1999), o que não precisa configurar problema, já que, se a cognição vem sustentar a linguagem, é porque ela tem estrutura lambda potencialmente infinita.

Tanto morfemas quanto palavras ou sintagmas terão suas especificidades sintáticosemânticas, e fonológicas. Um item lexical, então, vem para entrar na sintaxe com três informações: o conteúdo fonológico, o conteúdo sintático e a informação conceitual ou enciclopédica, ficando no formato < informação fonológica, árvore sintática, informação conceitual $>$. Os traços semânticos que comentamos acima - PATH e PLACE - estão na estrutura sintática. A informação conceitual, por sua vez, faz parte da herança enciclopédica e conhecimento de mundo que o item carrega.

De primeiro momento, a inserção lexical acontece por um mecanismo de compartilhamento (matching) entre o item e a sintaxe. Mas nem sempre um item é do mesmo tamanho da estrutura requerida pela frase. Ele pode ser maior ou igual, pode sobrar informação, que fica pressuposta na hierarquia funcional $(f-s e q)$. Logo, por princípio de superconjunto, pode sobrar informação, sempre em nós de cima, nomeada como lixo minimizado (minimise junk). Quando houver competição entre dois itens lexicais a ocupar uma posição na estrutura, Starke prevê duas etapas cíclicas. Ou seja, um entra na estrutura, mas há outro que também tenta. Nisso, se sobrar informações, o que tiver menos lixo ganha. Essa competição é nomeada como lexicalização cíclica.

Logo, os quatro princípios acima (compartilhamento, superconjunto, lixo minimizado e lexicalização cíclica) formam o conteúdo básico para explicar o que, em termos genéricos, nomeamos como 'lexicalização', 'codificação' ou 'inserção lexical'.

Vamos aos nossos dados.

\begin{tabular}{|c|c|c|}
\hline caitar & $\Rightarrow$ & {$[\mathrm{PLACE}]$} \\
\hline gankar & $\Rightarrow$ & {$[\mathrm{PATH}, \mathrm{PLACE}]$} \\
\hline
\end{tabular}

Num primeiro momento, vamos considerar um item lexical verbal que codifica PLACE, como 'caitar': 


\section{$=$ TRAMA $=$}

Nesses exemplos, o termo parece mesmo lexicalizar um evento estativo, posicional, que inclui uma determinada estratégia de jogo.

O verbo 'gankar', por sua vez, codifica PATH e PLACE:

(3) "ta gankando Kayn?"

(4) "Ivl 6 ou 7 eu te ganko ornn"

Intuitivamente, gankar parece trazer informações de um evento agentivo, também posicional, que inclui outra estratégia de jogo com movimento.

A $f$-seq verbal, na nanossintaxe (RAMCHAND, 2008), contém a hierarquia básica de traços de um iniciador (causa), de um processo e de um resultado de evento, estruturados na seguinte sequência: InitP, ProcP e ResultP, conforme Figura 2, Estrutura geral básica nanossintática para os verbos (adaptação de RANCHAND, 2008, p. 39).

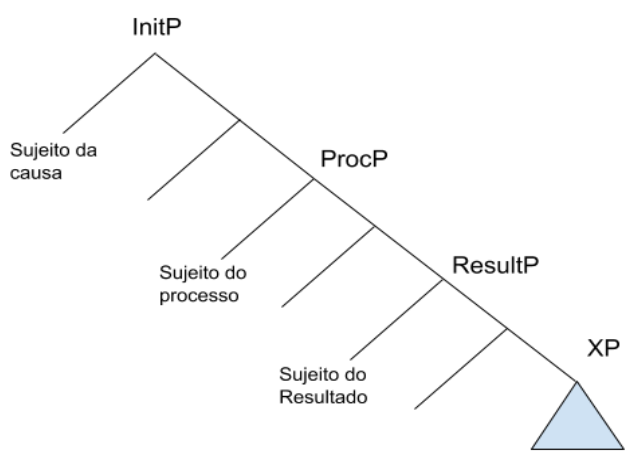

Estamos considerando, conforme a autora, que a estrutura acima seria a decomposição verbal que contempla uma estrutura causal, com InitP, ProcP e ResultP, e uma estrutura mais baixa em posição de complemento - genericamente representada por XP. XP pode ser, nesse sentido, um PathP, um PlaceP, ou mesmo um PathP seguido de um PlaceP (p. 111). Um verbo, portanto, pode conter uma combinação de traços, ou num fim último, a combinação total de todos os traços previstos na decomposição verbal. No processo de lexicalização, os nós de baixo são usualmente preenchidos por preposições, que introduzem os complementos verbais previstos em seu significado.

Um verbo achievement, como 'caitar', lexicaliza [ResultP]. Para além desse traço básico, caitar pede um conteúdo preposicional que codifica o PLACE, preenchendo o XP previsto na figura genérica de sequência verbal da figura 2. Convencionalmente, esse processo é representado pictoricamente pelo sinal " $\Rightarrow$ " (BAUNAZ \& LANDER, 2018), como na Figura 3 a seguir: 
Figura 3: Estrutura lexicalizada do verbo caitar

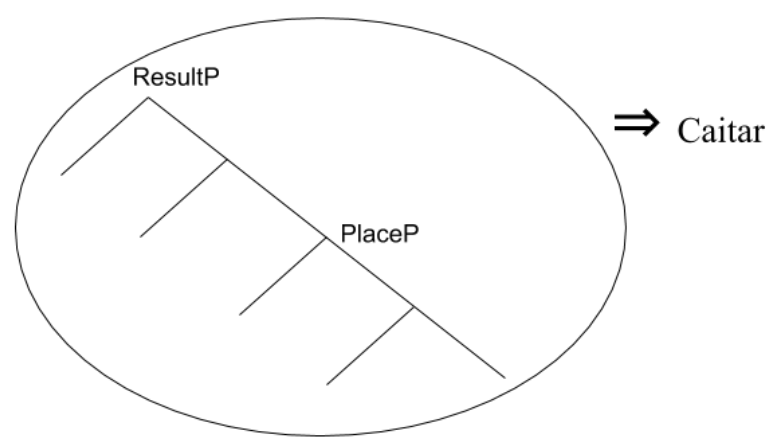

Fonte: Autoria Própria.

Com relação à hierarquia do conteúdo preposicional (que pode preencher $\mathrm{XP}$ ), Pancheva (2009), baseada em análise de mais de 80 línguas, propõe uma sequência genérica diagramada a seguir:

Figura 4: Hierarquia de traços espaciais lexicalizados pelas preposições (adaptação de PANCHEVA, 2009)

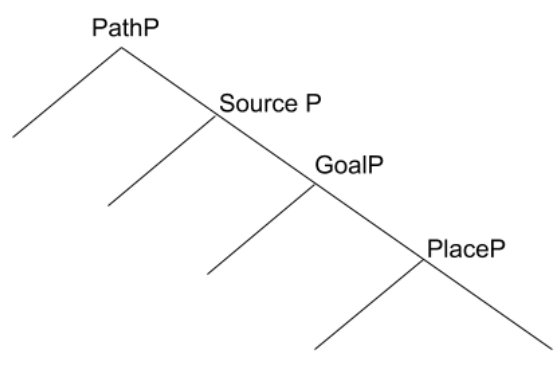

No caso do verbo 'gankar', um verbo atividade que codifica PATH e PLACE, a hierarquia de Pancheva é incorporada à estrutura do verbo:

Figura 5: Estrutura lexicalizada pelo verbo gankar.

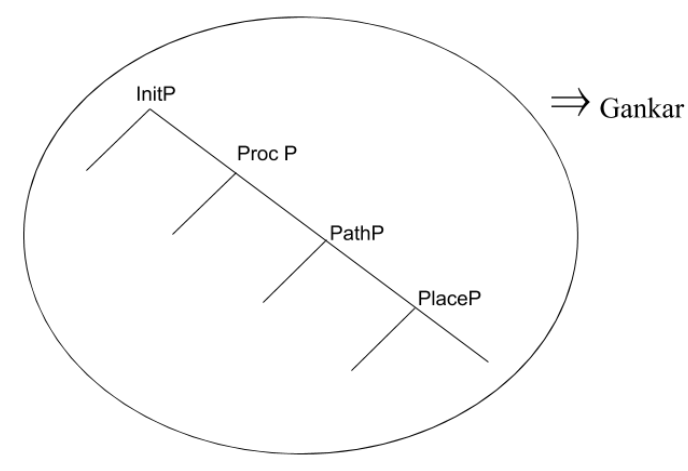




\section{$=$ TRAMA $=$}

Para checar a presença desses traços, a literatura em nanossintaxe (RAMCHAND, 2008; SVENONIOUS, 2010, apud FERREIRA, 2020) propõe alguns testes que captam as informações de PATH e PLACE como complementos. Um verbo como "estar", por exemplo, aceita complemento locativo (3):

(3) O jogador está embaixo da ponte.

Já um verbo como "cochilar" não precisa de complemento locativo; mas aceita sim adjuntos locativos preposicionados:

(4) O jogador cochilou feio embaixo da ponte.

As sentenças (3) e (4) revelam que o verbo 'estar' codifica PLACE', exibindo diacronicamente ainda os traços de localização espacial, e o verbo 'cochilar' não codifica PLACE, aceitando, portanto, modificação espacial sob o comportamento sintático de adjunto. Mas 'estar' não se comporta bem com complementos de trajetória (PATH). Logo, não seleciona PATH:

(5) ? O jogador está até a cerca.

O mesmo acontece com o verbo 'caitar':

(6) ? O jogador caitou até a ponte.

Por outro lado, 'gankar' aceita complemento PLACE e PATH:

O cara tá gankando até o inimigo na ponte.

Uma última observação nesta análise dos dados diz respeito à adaptação dos novos verbos à morfologia flexional do português brasileiro (PB). Os registros de falas dos gamers mostram que o léxico verbal novo adapta-se às operações gramaticais convencionais do PB sem restrições. Os verbos aparecem, e podem ser testados junto a informantes gamers, em valores aspectuais gramaticais diferentes (gankou, tá gankando), em tempos diferentes (vai gankar, tô gankando, fiquei gankando) e mesmo em valores modais distintos (pode gankar, tem que gankar).

$\mathrm{Na}$ estrutura dos blocos universais semânticos de Vonfintel \& Mathewson (2008), a gramática de valores semânticos de aspecto, tempo e modo está concentrada na segunda zona da derivação da figura 1. Em termos morfossintáticos, podemos também dizer que a morfologia funcional do PB atua sobre os itens novos sem restrições:

O que é conhecido como morfologia funcional incluí morfemas de ligação flexional assim como palavras funcionais livres como um, de, e, etc., isto é, todos os morfemas com alguma função gramatical. Todos os morfemas funcionais não mudam de categoria (SLABAKOVA, 2016, p. 58) ${ }^{8}$.

\footnotetext{
'O termo 'codificar', na nanossintaxe, se alterna com 'lexicalizar', mas parece registrar um sentido mais genérico do que o sentido de realização de estrutura semântico-sintática.

8 What is known as functional morphology includes inflectional bound morphemes as well as free functional words such as the, a, of, and, etc., that is, all morphemes with some grammatical function. All functional morphemes are noncategory changing.
} 


\section{$=$ TRAMA $=$}

Mas para o recorte a que optamos neste trabalho - os primitivos semânticos nos verbos novos dos gamers -, observamos que a estrutura nanossintática nos traz ferramentas para descrever hierarquicamente as combinatórias de traços básicos, que privilegiam, nesta investigação inicial, PATH e PLACE.

\section{CONSIDERAÇÕES FINAIS}

Neste artigo, nosso objetivo foi investigar a semântica de primitivos conceituais básicos que está sustentando a produtividade de verbos novos do jargão dos gamers, especificamente do jogo League of Legends ( $L O L)$. A análise evidenciou a recorrência de verbos que codificam o espaço, o movimento, a trajetória. Lançamos mão, então, de uma teoria que hierarquiza esses traços num léxico que compartilha sua estrutura com a sequência universal de traços sintaticamente organizados (f-seq). A nanossintaxe, uma das herdeiras dos pressupostos da semântica conceitual de Jackendoff, nos trouxe a opção representacional mais adequada.

Se o contexto de investigação fosse outro, talvez os traços mais produtivos também fossem outros. Num ambiente hospitalar, por exemplo, as sentenças enunciadas pelos médicos e outros profissionais de saúde talvez não exibam verbos que codificam o lugar e a trajetória. Talvez a lexicalização privilegiada fosse pelas informações de causa e de resultado, num discurso etiológico que a medicina privilegia. Este suposto contexto nos mostra que há de fato situações pragmáticas e discursivas que nos direcionam a outros padrões de lexicalização (Levin \& Rappaport-Hovav, 2019). Mas nossas conclusões nos levam a acreditar que há uma semântica de base que responde às diferentes frentes de comportamento linguístico. Uma semântica associada à sintaxe responde a essas questões com padrões de lexicalização baseados em estrutura universal.

\section{REFERÊNCIAS}

BARON, I; Herslund, M. Langues endocentriques et langues exocentriques: approche typologique du danois, du français et de l'anglais. Langue Française, 145, 2005, p. 35-53.

Baunaz \& Lander, 2018

BERTINETTO, Pier Marco. On a frequent misunderstanding in the temporal-aspectual domain: the 'perfectivetelic confusion. In: CECHETTO, C. et alii. Semantic Interfaces: reference, anaphora and aspect. Stanford:

CSLI Publications, 2001.

BOBALJIK, J. Distributed morphology. Ms, University of Connecticut., 2015.

CANÇADO, Márcia; GODOY, Luísa; AMARAL, Luana. Catálogo de verbos do português brasileiro.

Classificação verbal segundo a decomposição de predicados. V. 1 - verbos de mudança. Belo Horizonte: Editora UFMG, 2013

DIAS, M. R; RUTIQUEWISKI, A. F. Neologismo em League of Legends: uma análise pelo viés morfológico.

Trabalho de Conclusão de Curso, Universidade Tecnológica Federal do Paraná, Curitiba, 2019.

FERREIRA, Thayse Letícia. Uma investigação sintático-semântica das preposições espaciais do português brasileiro: uma abordagem nanossintática. Tese de doutorado, UFSCar, no prelo, 2020.

GRUBER, Jeffrey S. Studies in lexical relations. PhD, MIT, 1965, 310p.

JACKENDOFF, R. Semantics and cognition. Cambridge, M.A.: MIT Press, 1990.

JACKENDOFF, R. Meaning and the lexicon - the parallel architecture (1975-2010). Oxford University Press, 2012.

KRIVINE. Jean-Louis. A propos de l'intuition en mathématiques, 2018, https://www.irif.fr/ krivine/articles/Intuition.pdf, (acessado em 02/05/2019).

LANGACKER, R. Universals of construal. In: The annual Proceedings of the Berkeley Linguistic Society: General session and parasession on semantic typology and semantic universals, 1993, p. 447-463. LEVIN, Beth. English verb classes and alternations - a preliminary investigation. Chicago and London: The University of Chicago Press, 1993.

Levin, B.; Rappaport Hovav, M. Lexicalization patterns. In: Truswell, R. (Org.) The Oxford handbook of event structure. Oxford University Press, 2019.

LEVIN, B \& RAPPAPORT HOVAV, M. Building verb meanings. In: BUTT, M.; GEUDER, W. (Eds.) The projection of arguments: lexical and compositional factors. CSLI Publications, 1998, p. 97-134.

LEVIN, B \& RAPPAPORT HOVAV, M. Argument Realization. Cambridge: Cambridge University Press, 2005. 
RAMCHAND, Gillian; SVENONIOUS, Peter. Deriving the functional hierarchy. Language Sciences, n. 6, 2014, p. 1-37.

PANTCHEVA, Marina Blagoeva. Directional expressions cross-linguistically: nanosyntax and lexicalization. In: Nordlyd 36, 1 special issue on Nanosyntax. Svenonious, P; Ramchand, G. Starke, M.; Taraldsen, K.T. (Eds.), 2009, p. 7-39.

PUSTEJOVSKY, J. The syntax of event structure. In: Cognition, v. 41, p. 47-81, 1991.

PUSTEJOVSKY, J Decomposing Path: the nanosyntax os directional expressions. Tese de doutorado,

Universidade de Tromso, 2011, 301p.

PINKER, Steven. Learnability and cognition - the acquisition of argument structure. Cambridge, Mass: The MIT Press, 1989.

RAMCHAND, G.C. Verb meaning and the lexicon. Cambridge, 2008.

RAMCHAND, G.C. Situations and syntactic structures - rethinking auxiliaries and order English. The MIT Press, 2017.

RAMMÉ, Valdilena. A expressão do deslocamento nas línguas naturais: análise translinguística da estrutura [Vmaneira + Preploc]. Dissertação de Mestrado, Estudos Linguísticos/UFPR, 2012, 133p.

SAEED, John I. Semantics. Cambridge, MA: Blackwell, 2a. ed., 2003[1997].

SLABAKOVA, R. Second language acquisition. Oxford University Press, 2016.

SMITH, Carlota. The Parameter of Aspect. Dordrecht: Kluwer, 1997.

STARKE, M. Nanosyntax: a short primer to a new approach to language. Special issue on Nanosyntax.

Nordlyd, v. 36, n.1, 2009

TALMY, L. Toward a Cognitive Semantics. Vol. I e II. Cambridge, Mass: The MIT Press, 2001.

VENDLER, Z. Linguistics in Philosophy. Cornell University Press, Ithaca (NY), 1967.

VON FINTEL, Kai; MATTHEWSON, Lisa. Universals in semantics. In: The Linguistic Review, 25, p. 139-201, 2008.

WUNDERLICH, D. Cause and the structure of verbs. In: Linguistic Inquiry, 28, 1997, p. 27-68.

Recebido em 31-10-2020

Revisões Requeridas em 29-11-2020

Aceito em 14-12-2020 